Relations industrielles

Industrial Relations

\title{
James L. Gibson, John M. Ivancevich and James H. Donnelly Jr., Organizations: Behavior, Structure, Processes
}

\section{Alain Rondeau}

Volume 47, numéro 1, 1992

URI : https://id.erudit.org/iderudit/050754ar

DOI : https://doi.org/10.7202/050754ar

Aller au sommaire du numéro

Éditeur(s)

Département des relations industrielles de l'Université Laval

ISSN

0034-379X (imprimé)

1703-8138 (numérique)

Découvrir la revue

Citer ce compte rendu

Rondeau, A. (1992). Compte rendu de [James L. Gibson, John M. Ivancevich and James H. Donnelly Jr., Organizations: Behavior, Structure, Processes]. Relations industrielles / Industrial Relations, 47(1), 166-170.

https://doi.org/10.7202/050754ar

Tous droits réservés @ C Département des relations industrielles de l'Université Laval, 1992
Ce document est protégé par la loi sur le droit d'auteur. L'utilisation des services d'Érudit (y compris la reproduction) est assujettie à sa politique d'utilisation que vous pouvez consulter en ligne.

https://apropos.erudit.org/fr/usagers/politique-dutilisation/ 
much preferred small scale vertical unions which could serve as a communication device between labor and management. Thus the Kyochokai was created to put into place a model of industrial harmonism that represented such thinking. Ultimately this philosophic stance came to dominate the intellectual and ideological character of interwar labor relations in Japan.

While this philosophy manifested a concern for the worker and social reform, one senses a somewhat paternalistic approach which attempted to forestall protective legislation on the grounds that such legislation was unnecessary in a society where there was mutual respect and friendship in the workplace. Although the harmony of Japanese industrial relations is contrasted with the conflict evident in the West, there was never much doubt that Japanese management like its Western counterpart, intended to stifle traditional trade unionism and maintain control of its prerogatives. Although called by other names in Japan, one is reminded of various employee representation plans and other forms of welfare capitalism practiced in the West, during this same period of time. As a matter of fact, given the many cultural differences between Japan and the West, it is surprising how much overlap there was between the approaches to industrial relations in these very diverse parts of the world.

As to the question of whether harmony and cooperation were a function of ancient Japanese custom or a modern program put into place to assist Japanese industrialization, the author leaves little doubt. To quote Kinzley:

"What this study has shown, ... is that this tradition,... was consciously invented and propagated over the last century. It did not emerge of itself. The Kyochokai and earlier Meiji reformers, seeking to meet the situational needs of an emerging industrial society, were agents of its creation" ( $p$. 150).

Overall, this brief volume provides a good view of the role of the Kyochokai in Japanese industrial relations between the Wars. Aside from a few minor annoyances (not adequately defining some terms and insisting on periodically providing a transliteration from the Japanese for other terms), the reading of this book is a good investment of one's time.

Martin M. Perline

Wichita State University

Organizations: Behavior, Structure, Processes (7th. ed.), par James L. Gibson, John M. Ivancevich, and James H. Jr. Donnelly, Homewood, III., Richard D. Irwin inc., 1991, 774 p., ISBN 0-256-08046-1

La recension d'un volume de base en comportement humain au travail (organizational behavior) représente toujours un exercice un peu fastidieux et répétitif; la recette généralement utilisée pour l'élaboration des «textbooks» américains est bien connue et varie peu d'un auteur à l'autre. C'est, entre autres, ce pourquoi des revues comme Psychological Review ou Personnel Psychology regroupent et évaluent comparativement de tels ouvrages lors de leur parution. 
Toutefois, à la réception du volume de Gibson et al. un léger pincement au coeur m'a incité à y accorder une attention particulière; la première édition de ce volume (parue en 1973) avait été l'un de mes premiers ouvrages de référence en début de carrière, et je n'avais pas consulté les six éditions parues depuis (régulièrement à tous les trois ans!). II m'apparaissait intéressant de remonter virtuellement dans le temps pour comparer le traitement fait au comportement humain au travail par les mêmes auteurs à deux moments différents. Cela devrait mettre en évidence les transformations de la connaissance diffusée par un tel manuel en 18 ans et permettre d'évaluer sommairement si cette formule de réédition s'avérait un bon véhicule de mise à jour pour l'enseignement. II y aurait sûrement un apprentissage intéressant à en dégager.

La première constatation issue de cette comparaison tient déjà dans le titre lui-même. Alors qu'en 1973, le volume s'intitulait Organizations: Structure, Processes, Behavior, dans l'édition de 1991, l'aspect acomportement» (Behavior) apparait en premier lieu dans le titre. De fait, il semble que les auteurs ont conçu, au départ, un manuel portant sur l'étude de la gestion au sein des organisations ( $\times$...the study of managing within organisations» 1973, p. V) mais ce dernier s'est lentement transformé en ouvrage sur la gestion efficace du comportement humain au travail (*...effective management of organizational behavior... $* 1991$, p. V).. Cette première observation se confirme d'ailleurs lorsqu'on constate que la partie IV du volume original, traitant du comportement humain au travail (Behavior within Organizations), comprenait trois chapitres et comptait pour à peine $22 \%$ de l'ouvrage. Elle est maintenant placée au début de l'ouvrage et distribuée sur deux parties (Behavior within Organizations: The Individual et Behavior Within Organizations: Groups and Interpersonal Influence). De plus, elle comprend maintenant 10 chapitres et couvre $50 \%$ du volume.

La seconde constatation, plus importante cette fois, concerne la structure même du volume et sa pertinence pour véhiculer les notions contemporaines propres au champ du comportement humain au travail. $\hat{A}$ cet effet, l'analyse comparative met en évidence le fait que l'édition 1973 portait d'abord sur l'étude de l'organisation et de ses caractéristiques et s'intéressait en particulier à la contribution apportée par les sciences du comportement à la connaissance sur ce sujet. Dans cette perspective, il était alors logique d'adopter en quelque sorte un point de vue managérial et de s'intéresser d'abord aux structures et aux processus organisationnels pour ensuite explorer les phénomènes comportementaux susceptibles d'influencer l'organisation du travail. Au contraire, l'édition 1991 reconnaît d'emblée l'existence d'un corps de connaissance propre au comportement humain au travail et entreprend de présenter la contribution de cette science à la connaissance des organisations. La perspective d'analyse est complètement changée et pourtant les auteurs ont conservé la même structure au volume, ce que met en exergue l'anachronisme de la formule de réédition pour refléter adéquatement l'évolution de la connaissance propre à un champ d'activité.

Au delà de cette comparaison globale toutefois, l'édition de 1991 mérite une analyse plus détaillée.

L'introduction (Part I) comprend deux chapitres. Au premier, intitulé The Study of Organisations, les auteurs insistent sur l'importance de l'étude du 
comportement humain au travail et précisent, comme en préface, l'apport des chapitres suivants à la compréhension de ce phénomène. Dans l'édition originale de 1973, le premier chapitre, portant le même titre, traitait plus pertinemment, il semble, de l'utilisation de la méthode scientifique dans l'étude des phénomènes organisationnels. L'édition de 1991 relègue maintenant cette information (vaguement mise à jour) en annexe (Appendix A). Le second chapitre, intitulé: Managing Individual, Group and Organisational Effectiveness présente une brève revue des courants d'étude de l'efficacité organisationnelle et en suggère un modèle synthèse intéressant pour guider l'action du gestionnaire. Ce chapitre se termine assez étrangement par une présentation du concept de culture organisationnelle et de son impact potentiel sur l'efficacité de l'organisation. Ce sujet apparaît ici, semble-t-il, comme un ajout imprévu et illustre bien le traitement pénible d'une préoccupation nouvelle dans le cadre d'une structure déjà déterminée.

La seconde partie (Behavior within Organisation: The Individual) et la troisième partie (Behavior within Organisations: Groups and Interpersonal Influence), couvrent au total 10 chapitres et constituent, somme toute, le coeur de l'ouvrage. On y retrouve les connaissances de base en comportement humain au travail selon un traitement classique, commun à ce type de manuel. II s'agit d'un travail honnête présentant de façon descriptive et bien illustrée les notions les plus couramment utilisées dans l'enseignement du comportement humain au travail et leur application à des situations de travail.

Après un chapitre (Chapter 3 - Individual Behavior and Differences) essentiellement axé sur la définition des variables individuelles affectant le comportement humain au travail, les deux chapitres suivants portent sur la motivation au travail et ses applications. Le chapitre 4 présente d'abord les théories dites du «contenu» de la motivation, puis, au chapitre 5, suivent les théories dites du aprocessus» de la motivation, reprenant en cela une classification suggérée il y a plus de 20 ans par Campbell, Dunnette, Lawler et Weick (1970) et déjà utilisée dans l'édition de 1973. Notons que l'emploi de cette formule a toutefois l'avantage de permettre le traitement de nouvelles théories sans modifier la structure de l'ouvrage!

La partie II comporte en outre deux autres chapitres. Le premier, à caractère résolument appliqué (Chapter 6 - Evaluating, Rewarding, and Punishing Behavior), semble être situé au mauvais endroit dans l'ouvrage. II porte sur les outils et pratiques utilisés en gestion des ressources humaines pour traiter des questions d'appréciation du rendement, de rémunération et de discipline en organisation. Ce chapitre trouverait sûrement mieux sa place dans un manuel traitant des questions de apersonnel». Le dernier (Chapter 7 - Stress and the Individual) reprend une teinte théorique et comportementale cohérente avec les chapitres précédents. II traite fort adéquatement du stress et de ses conséquences tant individuelles qu'organisationnelles et des programmes de gestion du stress.

La partie III conserve cette même formule visant à bien définir les concepts traités et à en clarifier l'impact sur le comportement humain au travail. Le chapitre 8 (Group Behavior) couvre relativement bien l'ensemble des notions de groupe les plus fréquemment utilisées. Le chapitre 9 (Intergroup Behavior and Managing Conflict) analyse le phénomène du conflit en organisation mais de 
façon très limitée. De fait, il est heureux qu'il en soit ainsi puisque les notions de leadership et de pouvoir n'ont pas encore été traitées. Au chapitre 10, on retrouve justement ces notions de pouvoir et d'exercice du jeu politique en organisation. Enfin, les chapitres 11 et 12 traitent des questions de leadership en respectant, encore une fois, une démarche plutôt conventionnelle. Après une brève présentation du concept de leadership (mal distingué des concepts d'autorité ou de pouvoir), le chapitre 11 expose les contributions apportées à la connaissance en leadership par les théories des traits, les théories normatives et situationnistes sur la question. Le chapitre 12 présente, par la suite, les courants un peu plus récents en leadership, notamment la théorie de l'attribution, le leadership charismatique ou le leadership transformationnel. Toutefois, comme c'était le cas en motivation au travail (chapitre 4 et 5), on adopte ici une approche additive à la connaissance. En effet, la structure du manuel rend possible l'addition d'une nouvelle contribution sans véritablement tenter d'effort intégrateur.

La partie IV (The Structure and Design of Organizations) reprend essentiellement ce qui était couvert à la partie II de l'édition 1973. En définitive, les notions purement administratives présentées ici semblent sensiblement les mêmes que dans cette première édition et, partant, risquent d'être moins appropriées que ce que l'on pourrait trouver dans un manuel récent d'administration. La valeur principale du traitement que l'on en fait ici vient de ce qu'on les considere en rapport avec leurs effets sur le comportement humain au travail. De toute évidence toutefois, cette partie est moins intéressante que la précédente.

Elle comprend trois chapitres (Chapter 13, 14, 15). Le premier présente divers concepts de base en structuration de l'organisation (Organization Structure). II prend sa réelle valeur dans les quelques pages de réflexions qu'il propose concernant l'effet du niveau de formalisation, de centralisation et de complexité de la structure organisationnelle. Le second, sur l'aménagement des tâches (Job Design), est essentiellement orienté sur les travaux ayant pour objet l'amélioration du potentiel d'une tâche à satisfaire son titulaire. Les travaux traités ne vont pas au delà des préceptes de l'approche socio-technique. Enfin, le dernier, sur l'aménagement organisationnel (Organizational Design), ne présente que des données classiques sur la relation entre la structure, le type de technologie et le niveau d'incertitude de l'environnement.

La cinquième partie, portant sur les processus organisationnels (The Processes of Organizations), n'apparaît pas, elle non plus, comme particulièrement innovatrice. Le chapitre 16 , traitant de communication, dépasse, certes, les préoccupations purement descriptives de l'édition 1973 mais n'est centré que sur les quelques suggestions classiques permettant généralement d'améliorer l'efficacité de la communication organisationnelle. Le chapitre 17, portant sur le processus de prise de décision, n'a subi que peu de transformation par rapport à l'édition originale et présente des généralités sans grand intérêt. Enfin, le chapitre 18, porte sur les processus de socialisation et de carrière, une préoccupation qui n'existait pas dans l'édition de 1973. Même s'il semble légitime de parler de socialisation comme d'un processus organisationnel, il n'est pas évident qu'il soit approprié de considérer la carrière individuelle dans ce cadre. II aurait peut-être été plus intéressant (et plus 
naturel) de regrouper ici les thèmes de socialisation et de culture organisationnelle.

Enfin, la sixième partie (Developing Organizational Effectiveness) semble souffrir, elle aussi, de stagnation et ne présente que peu d'éléments nouveaux ou dignes de mention. Des trois chapitres qu'elle comptait en 1973, l'édition de 1991 est réduite à deux chapitres qui traitent essentiellement du processus d'intervention en matière de développement organisationnel (Chapter 19 Organizational Development: Improving Performance) et des types d'interventions disponibles (Chapter 20 - Organizational Development Interventions). II ne reste malheureusement plus rien des préoccupations concernant le aclimat organisationnel» ou le adiagnostic organisationnel» de l'édition originale.

En somme, la valeur de cet ouvrage tient à sa transformation, malheureusement incomplète. D'un manuel hybride (management et comportement), il a été lentement mais définitivement transformé en manuel de base sur le comportement humain au travail. C'est grâce aux parties II et III qu'il prend vraiment sa valeur et que sa contribution devient appréciable. Les parties IV, $\mathrm{V}$ et VI ne semblent avoir été maintenues que pour conserver l'intégrité de la structure originale du volume et ne contribuent à l'ouvrage que parce qu'elles documentent marginalement l'effet de la structure ou des processus organisationnels sur le comportement humain.

II convient toutefois de souligner la valeur pédagogique de cet ouvrage, truffé d'exemples pratiques, de cas et d'exercices, de sommaires et de questions synthèses, de références et de notes bibliographiques. Ce manuel est conçu pour répondre aux préoccupations de tout jeune professeur (voilà pour ma nostalgie!) en quête d'une connaissance organisée, de définitions claires et d'illustrations appropriées.

Enfin, il importe de remarquer que la structure même du volume favorise une mise à jour à caractère additif. Certes, cette formule ne favorise pas l'émergence de synthèses nouvelles ou d'intégration originale de la connaissance mais ce n'est pas là, il semble, la mission de ces manuels. Comme outil pédagogique, cet ouvrage a sûrement sa place parmi tant d'autres semblables.

Alain Rondeau

École des Hautes études commerciales Montréal

Sachez évaluer votre personnel: le chemin de la réussite, par Denis Boucher et Christian Doyon, Montréal, Les Éditions Agences d'Arc, 1991, 180 p., ISBN 2-89022-254-3

L'évaluation du personnel peut se décrire comme un processus qui identifie, observe, mesure et développe la performance humaine dans les organisations. La mesure de la performance au travail a été un sujet d'intérêt et un champ d'investigation depuis l'apparition de la psychologie 